\title{
Human-Robot Interaction System Research for 500kV EHV Power Transmission Line Inspection Robot
}

\author{
Weibin Guo ${ }^{1,2, a}$, Hongguang Wang ${ }^{1, b}$, Peng Sun ${ }^{1, c}$ and Lie Ling ${ }^{1, d}$ \\ ${ }^{1}$ State Key Laboratory of Robotics, Shenyang Institute of Automation (SIA), Chinese Academy of \\ Sciences (CAS), Shenyang 110016, PRC \\ ${ }^{2}$ Graduate School of the Chinese Academy of Sciences, Beijing 100049, PRC \\ âweibing@sia.cn, bhgwang@sia.cn , 'sunpeng@sia.cn, 'linglie@sia.cn
}

Key words: Power line inspection robot, Tele-robotics, Human-Robot Interaction, software design

\begin{abstract}
An assisted and secure interaction system is developed for $500 \mathrm{kV}$ EHV Power Transmission Line Inspection Robot. The challenges on amenity, assistance and security of interactive operation are emphasized. Due to the unpredictable line structure and the incomplete sensory, the robot is controlled in remote and locally autonomous mode. Based on the Finite State Machine (FSM), the automatic crossing interface is designed for protecting operators from memorizing redundancy sequences. Measures for friendly operating and avoiding errors are concerned. The architectures for fully autonomous are also engaged. The lab and field experiments validated the interaction system reliable and easy to use.
\end{abstract}

\section{Introduction}

Recently, power line inspection and maintenance robot research has become a focus ${ }^{[1]}$ of the robotics at home and abroad. The inspection robot was firstly researched since 1980s. Then, the LineScout and Expliner were developed respectively in Canada ${ }^{[2]}$ and Japan ${ }^{[3]}$. From the 1990s, Wuhan University ${ }^{[4]}$, Shenyang Institute of Automation (SIACAS) ${ }^{[5]}$ and Institution of Automation ${ }^{[6]}$ pursued the inspection robot independently. SIACAS has been devoting themselves to the power line inspection and repair robots since 2002. In 2003, AApe- $A{ }^{[7]}$ was manufactured for one span. In 2006, AApe-B was developed for one strain section. In 2009, AApe-B3 was improved for the whole path. Now, we are designing the newest type $A A p e-D$ for broken strands repair.

A complete human-robot system consists of operators, the robot, Human-Robot Interface (HRI) and environment. The HRI research ${ }^{[8 \sim 11]}$ focus on interaction paradigms and design models, human computer adaptation, usability evaluation, multi-modal technique, and natural interaction. The Ground Control Station (GCS) interface ${ }^{[12,13]}$ of Unmanned Aerial Vehicle (UAV) focuses on flight control, real-time display and route plan. The trends are for the universal interface, intelligent decision and security techniques. A mine rescue robot interface ${ }^{[14]}$ is researched with motion control and monitor for robot and environment. The task complexity and operating security are the key challenges for overhead inspection robot.

The Line Scout interaction ${ }^{[2]}$ strategy of intuitive control, safety protection, assisted operation and open architecture are researched, while obstacles and faults detection are not included. Wuhan University robot ${ }^{[4]}$ interface focuses on fault detection and database construction. The Explainer interface ${ }^{[3]}$ is for the robot monitor and control, while information processing is not mentioned.

According to the cooperation principle between operators and inspection robot, an interface concerned with the amenity, assistance and security is proposed for AApe-B3. A clear layout is devised for state monitor and intuitive control. The automatic crossing interface is designed worthy for assisted operation with improved security and efficiency. The interaction system is friendly interactive and simple to use. 


\section{Overview of the robot system}

The prototype of the robot. In Fig. 1 (a), the robot contains two arms, a slide, and a cabinet. The fore arm includes walking system, prismatic and revolute joints, driven by MOV1, GRP1, LIF1 and ROT1. CAM1 and CAM3 are fixed at the fore arm for motion monitor. The rear arm is identical and configured dissymmetrically. The dual arms distance and centroid adjusting mechanism are on the slide, driven by TRA and EQU independently. The Pan and Tilt Camera (PTC) is used for detailed inspection.

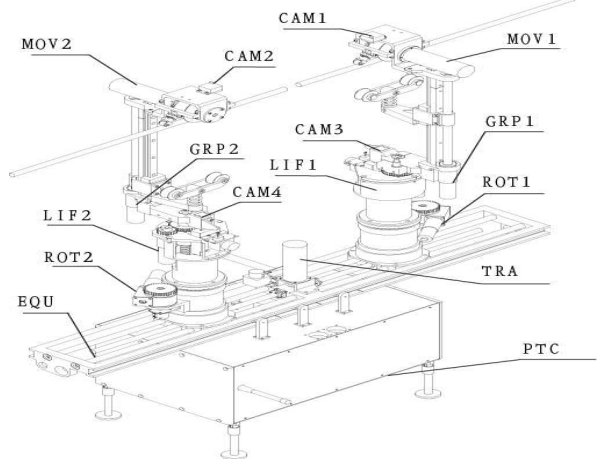

(a) Mechanical configuration

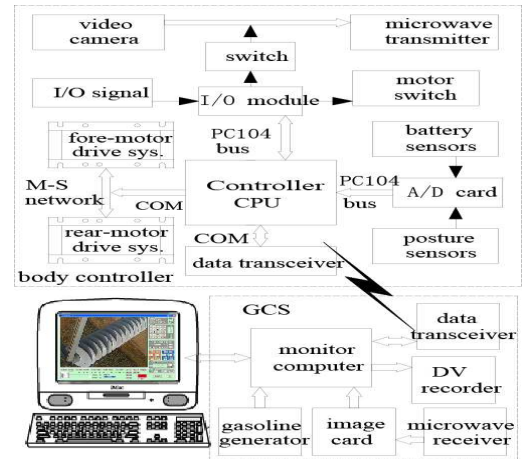

(b) Control system

Fig. 1. Mechanism and Control system of AApe-B3

In Fig. 1 (b), the control system contains body controller and GCS. The body controller is powered by rechargeable lithium battery of $24 \mathrm{~V}, 20 \mathrm{Ah}$. The motor controllers are grouped by serial network. The GCS includes a monitor computer, a ground case, antennas, and a gasoline generator. The ground case is housed with the data radio, microwave receiver and digital video recorder. Data radio is connected with the computer by COM, and microwave receiver is connected by the image card. Moreover, the user interface is running on the computer.

Remote and locally autonomous control. The GCS and robot communicate wirelessly. The robot works in remote and locally autonomous mode. The robot can walk along the power lines by wheels rolling, and cross obstacles by negotiation mechanism. With tool modules, the robot can perform repair jobs.

The robot works on live-lines 40 meters high above the ground. Un-modeled field environment and unknown obstacle postures and sequences increase the barriers for control. Besides, incomplete sensory makes it useful for operators intervene with the automatic motion. According to the specialty of humans and the robot, the task for human control and autonomous motion should be assigned properly. The automatic crossing interface is designed to greatly facilitate the crossing operation.

\section{Interaction system design}

Remote control requirements

Remote Monitoring

--Environmental Monitoring. The visual method is usually applied for environmental monitoring. The monitor for running surroundings and line defects inspection are included. The robot motion image and inspection image should be sent back for monitoring.

--Robot Pose Monitoring. Robot angle, obstacle signal, walking speed and grippers' state, joints data and centroid position are included. The robot pose should be monitored for motion control.

--Robot Status Monitoring. The load voltage and current, battery state of charge, angle alarm and communication state are included. The robot running status should be monitored for safety.

\section{Motion Control}

--Direct Motion Control. The robot actions control is included.

--Attitude Adjusting Control. The control for joints reposition, cabinet horizontality, combined motion and reconfiguration are included.

--Automatic Crossing Control. The obstacle types include the compression pipe, counterweights, suspension clamps and the subsidiary track for strain tower. 
--Image Scouting Control. The PTC viewing direction and turning speed can be controlled. The channels for inspecting and monitoring can be switched. The inspection image can be zoomed, and the aperture and focus can be controlled. The image can be captured to picture or recorded to video.

\section{Information Management}

--Robot Data Management. The model and the kinematics data should be stored for motion simulation and control. The cell motion, robot pose and state, obstacle patterns and wheel-line alignment image should be included for motion plan and safety diagnosis.

--Task Data Management. The data for transmission line, defects pattern and suspected defect image should be handled for intelligent detection and maintenance record.
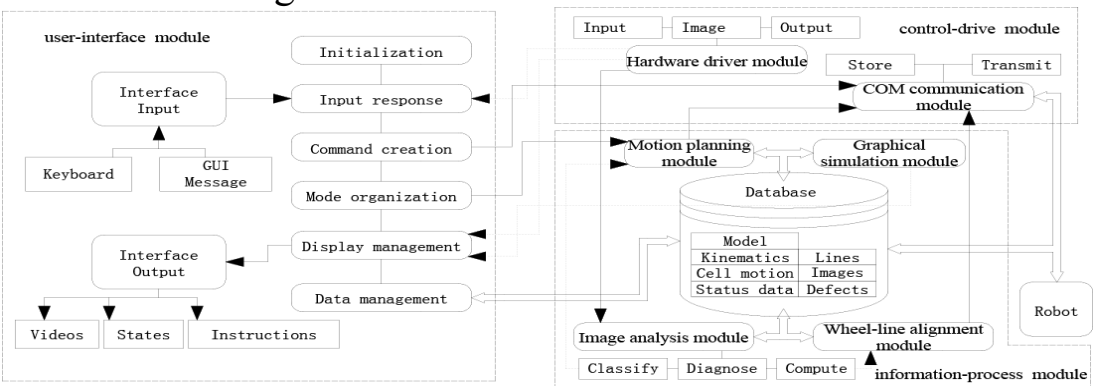

Fig. 2. The human-robot interaction system software construction

Software design. In Fig.2, the interaction system includes three parts as user-interface module, control-drive module and information-process module. The first is used for humans interacting with the computer, including main interface, auxiliary interface, and automatic crossing interface, in Fig.3 (a). The second contains hardware driver and COM communication. The third contains image analysis, graphical simulation, motion planning, wheel-line alignment and database.

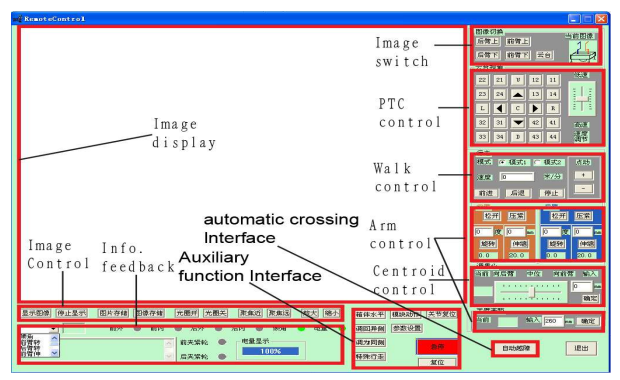

(a) Main interface

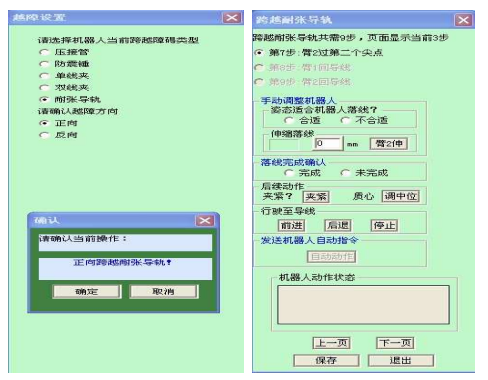

(b) Automatic crossing interface

Fig. 3. User Interface

\section{User-interface module design}

In Fig.3 (a), main interface consists of motion function region, image function region and information function region. The first is at the right middle. The walk control, arm control and centroid control are included. The second is at the right top and left middle. Channels switch, PTC control and the image operation are included. The third is primarily at the left side. The image display, sensor query, action state and pose feedback are included.

In Fig.3 (a), the auxiliary interface is at the lower part. Emergency stop, parameters setup and attitude adjustments are included. The parameters include arms action step, robot speed and clamping current etc. Attitude adjustments contain cabinet horizontality, combined motion, joints reposition and reconfiguration etc. The combined motion contains two arms joint rotating and joint lifting, and the gripper and arm joint action etc. The joints reposition includes actions of arms and centroid etc. Reconstruction is used for mechanism configuring in crossing the subsidiary track of strain tower.

In Fig. 3 (a), the automatic crossing interface is configured at the right bottom. In Fig.3 (b), the obstacle crossing choice is shown in the left. The 7th step for crossing the subsidiary track is shown in the right of Fig.3 (b). The subsidiary track crossing contains nine steps. Each step is constructed by a dialogue page with three aspects planning; the first is wheel-line alignment; the second is posture adjustment; and the third is automatic motion. The control and feedback status is shown on the rectangle at the bottom. The operator can be instructed step by step to complete the obstacles crossing. 


\section{Control-drive module design}

\section{1) Hardware driver design}

As shown in Fig.2, the image capture card is driven for video record and image operation. The digital I/O card is driven for control signal input and display signal output.

HANDLE okOpenBoard(long*iIndex) and BOOL okCloseBoard(HANDLE hBoard) are called for operating the image card. The image storage and display are programmed by Long okSaveImageFile(HANDLE hBoard, LPSTR szFileName,long first,TARGET target,long start,long num) and BOOL okCaptureTo(HANDLE hBoard, TARGET target,LONG wParam, LPARAM 1Param).

The digital I/O card is driven by HANDLE DeviceOpen() and BOOL DeviceClose(HANDLE m_DeviceHandle). The input and output are achieved by int ReadInput(int InputN) and BOOL WriteOutput(int OutputN,int OutputValue).

2) COM communication design

The COM is used for communication between GCS and the robot. The sending commands and receiving data are recorded for planning and diagnosis, as shown in Fig.2.The MSComm object is used for receiving. The COM status is obtained by GetCommEvent().GetInput()is called for receiving data. The sending thread UINT CGlobalCOM::SendDataThread(LPVOID pParam) is programmed running concurrently with the main thread.

\section{Information-process module design}

1) Image analysis design

The image analysis, used for motion planning and defects detecting, is primarily composed of classifying, diagnosing and computing, as in Fig 2. The general image processing methods, including gray scale processing, noise filtering and image segmenting are adopted. Based on geometry features, the eigenvectors are extracted for objects classifying, such as counterweights, insulators or suspension clamps. The objects classifier provides targets for motion planning and defects detecting. The defects classifier is used for fault identification. Moreover, the deviation for wheel-line alignment control can be computed from the motion image.

2) Graphical simulation design

The robot real-time motion displaying, is useful for intuitive operation. With posture sensors feedback, the simulation is performed simultaneously. Modeled in SolidWorks, the motion display is designed with OpenGL.

3) Motion planning design

The motion planning is used for data fusion and inference. According to lines data and the image classifier result, the proper action mode is voted for motion control. The action modes, stored in the database, are designed as motion sequences and combined motion. By an inference system, the evaluation of the robot state of health and safety is obtained from the battery state and joints pose. Then the next action is locked or secured.

Ape32.dll is developed for motion control interface. BOOL SetRobotMotion (long Motiontype, long Motiondata, bool Motionid, int Motionsn) is used for joints control. The locally automatic crossing is achieved by bool SetTask (long ObjectType, long OpenAngle, long LiftData, bool Motionid, Int TaskSN). VOID SetPan_Tilt (intCameraID, int PosID) is used for PTC control. VOID GetSensorData (long SensorID) and bool SensorDecode (long\& SensorID, double\& Sensordata, bool \&IOdata) are used for sensor query. BOOL StateCheck (long\& Motiontype, bool\& ActId, bool\& ActState) is used for action state checking. BOOL SetHandShake (long StartID) is used for handshaking.

4) Wheel-line alignment design

The robot kinematics and the control algorithm are included in the wheel-line alignment. With the deviations computed by the motion image, we established the control model. The un-calibrated visual servoing method is applied for wheel-line alignment control. The neural network schema is used for constructing the control model between the image space and the Cartesian space. 
5) Database design

In Fig.2, the robot model, kinematics, cell motion, lines structure, captured images, defects data and robot state data are included in the database.

Mode of automatic sequences. The robot parameters are shown in Table 1. The dual arms distance is $260 \mathrm{~mm}$, and the robot is going upgrade of 10 degrees. Based on two basic crossing modes ${ }^{[6]}$, we designed the automatic crossing sequences. In the rotation mode, the wheel motors MOV1-2 are disabled. In the cankerworm mode, the arm motors ROT1-2 are disabled.

Table 1 Robot joint Parameters

\begin{tabular}{ccccc}
\hline Joint type & Arm prismatic & Arm revolute & Arms distance & Box prismatic \\
\hline Variable limit & $20 \sim 180 \mathrm{~mm}$ & $-50^{\circ} \sim 220^{\circ}$ & $260 \mathrm{~mm} \sim 420 \mathrm{~mm}$ & $-400 \mathrm{~mm} \sim 400 \mathrm{~mm}$ \\
\hline
\end{tabular}

Communication protocol design. The protocols include bidirectional commands between GCS and the robot, such as handshake, emergency stop, control and feedback. The handshake format is identical, and emergency stop has highest priority. Others are common for control and feedback, as follows.

The protocol contains 15 characters. The first indicates the start bit of a full command. The last is used as end detection. The second is used as the command types, such as motor test, action or camera control, combined motion, automatic crossing, and sensor query. The 3rd and 4th are used as the command objectives, such as IDs for motor, camera or arms, walk mode and crossing direction. The 5 th and 6th are used for motion index, such as motor mode, IDs for actions, obstacles, PTC poses or sensors. The 7th to 12th are used as data for actions or sensors. The 13th and 14ths are used as checksum or state feedback.

\section{Validation and field experiments}

The former interface is shown in Fig.5 (a). Then, the interface is improved as follows. The popup dialogue positions are specified for not interrupting the video display and other control buttons. The layouts for frequently used function are adjusted according to the human physical characteristics. The data for sensors and control of the former interface are very hard to understand. Therefore, the control data of walking speed in $\mathrm{m} / \mathrm{min}$., the displacement in $\mathrm{mm}$ and the rotation in deg are used. Sensors data in deg, mm, Ampere, Voltage and percentage are used. The confirm dialogue is designed for preventing error operations in a clear way. Other techniques are applied for simplifying the operator control, such as icons, the slider, combo box and the process. The layout and the color scheme are evaluated, and improved repeatedly to make the functional layouts clear and easy for dividing, as in Fig.3 (a). At last, the operating consolidation and convenience are validated.

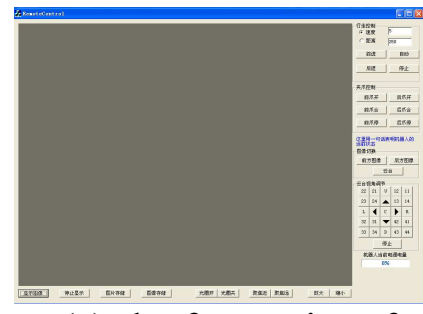

(a)The former interface

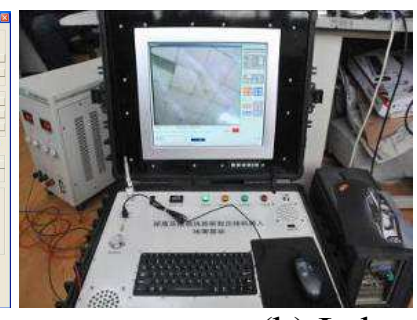

(b) Lab and Field experiments

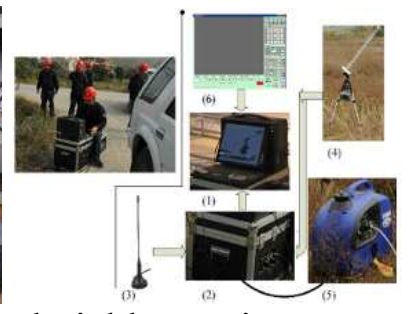

Fig. 5. Validation and Experiments

The control-drive module is tested especially on the COM communication module. We designed the tests between two industrial computers connected by serial COM. The interface is running on one of the computers, while the serial debugging assistant is running on the other computer. The control commands are organized by the interface. When buttons are pushed, the commands are shown on the interface, while the sending results are monitored by the other computer. Compare the two results to 
validate the sending function. The feedback data Interpretation is validated by similar methods. The software platform for image process is preliminarily constructed and the database is designed. The robot control experiments in Fig.5 (b) validate the interaction system available.

\section{Conclusion and future work}

An interaction system featured with clear layout, legible supervisory and secure operation is achieved. The system architecture is constructed and software designing methods are depicted detailedly. Based on automatic sequences, we designed the automatic crossing interface in remote and locally autonomous mode. The proper protocols are designed, and the whole interaction system is developed. The lab and field experiments validate the convenience and reliability. The future work will focus on exploring the image recognition and visual control schema suited for field and nature environment; as well as the application of the automatic navigating and intelligent detection.

Manuscript received June 29, 2011. This work was supported in part under Grant 60875082.

\section{References}

[1] Toussaint, K, Pouliot, N., and Montambault, S., "Transmission Line Maintenance Robots Capable of Crossing Obstacles: State-ofthe-Art Review and Challenges Ahead", Journal of Field Robotics, vol. 26, no.5, 477-499, Wiley.

[2] Pouliot, N. , Latulippe, P. , and Montambault, S. Reliable and Intuitive Teleoperation of LineScout: a Mobile Robot for Live Transmission Line Maintenance. In: Proceedings of The IEEE/RSJ International Conference on Intelligent Robots and Systems, St. Louis, USA, 2010: 1703-1710.

[3] Debenest , P., Guarnieri, M., Takita, K., Fukushima, E., Hirose, S., Tamura, K., Kimura, A., Kubokawa, H., Iwama, N., \& Shiga, F., Morimura, Y. and Ichioka, Y. Expliner - Toward a Practical Robot for Inspection of High-Voltage Lines. Field and Service Robotics 7, STAR 62, 2010: 45-55.

[4] Wu G P, Xiao X H, Xiao H, Dai J C, Bao W J, Hu J. Development of a Mobile Inspection Robot for High Voltage Power Transmission Line. Automation of Electric Power Systems, 2006, 30(13):90-93.

[5] Wang H G, Jiang Y, Liu A H, Fang L J, and Ling L. Research of Power Transmission Line Maintenance Robots in SIACAS. In: 1st International Conference on Applied Robotics for the Power Industry, Delta Centre-Ville Montréal, Canada, 2010: 1-7.

[6] Fu S Y, Hou Z G, Liang Z Z et al. Image-based visual servoing for power transmission line inspection robot. International Journal of Modelling, Identification and Control, 2009, 6(3):239-254.

[7] Tang L, Fang L J, Wang H G, Zhang H Z. Inspection Robot Control System of Power Transmission Line Based on Distributed Expert System. ROBOT, 2004, 26(3):267 271.

[8] Dong S H. Progress and Challenge of Human-Computer Interaction. JOURNAL OF COMPUTER AIDED DESIGN \& COMPUTER GRAPHICS, 2004, 16(1).

[9] Wang H A, Dai G Z. human-computer natural interaction technology. Journal of Image and Graphics, 2010,(7).

[10] Agah, A. Human interactions with intelligent systems: research taxonomy. Computers and Electrical Engineering, Vol. 27, No. 1, January 2001, 71-107.

[11] ACM SIGCHI (1992). Curriculum for Human-computer Interaction. ACM Special Interest Group on Computer Human Interaction Curriculum Development Group, New York.

[12] Si B, Zhou C Z, Yu G. Design and Realization of Monitor and Control Base Station Software for MICRO-UAV. System Simulation Technology, 2007, 3(02):90-95.

[13] Zhou Y. A Review of UAV GCS Development. AVIONICS TECHNOLOGY, 2010, 41(01):1-6.

[14] Hou X L, Zhang Y F, Li X D, Sun J, Li Q. Design of the Supervisory Platform for a Mine Rescue Robot. SHANDONG SCIENCE, 2009, 22(05):68-71. 